\title{
CRACKING PHENOMENA OF COATINGS ON LABEL PAPERS
}

\author{
KAJA BRESKVAR, JURE AHTIK and KLEMEN MOŽINA \\ University of Ljubljana, \\ Faculty of Natural Sciences and Engineering, Department of Textiles, Graphic Arts and Design, \\ Snežniška ulica 5, 1000 Ljubljana, Slovenia \\ ๔ Corresponding author: K. Možina, klemen.mozina@ntf.uni-lj.si
}

Received February 3, 2021

\begin{abstract}
The phenomenon of paper coating cracking when folded was studied. Coating mixtures were compared according to their composition, applied on different base paper, to find which combinations of the constituents of the base paper and the paper coating results in a lower tendency towards fold cracking of the coating layer. It was shown that the appropriate selection of pigments (calcium carbonate, kaolin) and binders (starch, latex), whose elastic behavior extensively influences the deformation of paper under physical and mechanical stress, is of central importance. The choice of the base paper plays a significant role as well, it should have the longest and the most numerous cellulose fibers possible in order to avoid fiber breaks and inter- and intra-fiber connections. The content of inorganic substances has the greatest negative effect on deteriorating the physical-mechanical properties of the studied label papers, leading to the deposition of inorganic substances at the joints between the cellulose fibers and reducing their bonding. ${ }^{1}$
\end{abstract}

Keywords: label paper, coating, coating cracking, paper folding

\section{INTRODUCTION}

High print quality requirements often lead to the choice of coated paper, which offers better surface quality and can reproduce a wider color gamut than uncoated paper. Coating paper is a finishing process that improves, for example, optical and printing properties, while impairing physical-mechanical properties. The aim of paper folding is to bring the printed product into a form that can be directly processed or reproduced in order to carry out a further finishing process. ${ }^{2-5} \mathrm{In}$ order to avoid line cracks when folding the paper, it is necessary for the paper to have the properties of layering and even distribution of forces on the outside. ${ }^{6}$ The defect at the buckling point turns out to be a line that is usually parallel to the folding line; however, it may be oriented differently. ${ }^{7}$ Paper coating cracking is manifested in a fibrous paper layer, in which the bonds between the fibers are disconnected, and in some cases, even the cellulose inter-fiber network is broken, resulting in a loss of tensile strength. The negative effect is also reflected in the appearance of the final product, as it leads to a number of complaints that manufacturers want to avoid. ${ }^{8,9}$

Coated papers must have the capacity of an elastic body, which is largely influenced by the choice of components of the coating. The selection of pigments depends on the magnitude of paper deformation. Calcium carbonate is more appropriate for higher loads, as $\mathrm{CaCO}_{3}$ particles tend to have a round, isometric shape and therefore no fiber damage occurs, whereas kaolin particles have a flat shape that damages fibers more quickly. On the other hand, a kaolin-based coating provides better resistance against paper coating cracking during bending, when the load is applied axially on the kaolin particles. Starch is commonly used in paper production, i.e., in paper coating mixtures, due to its low price. However, because of its low elastic properties, thus-coated papers present higher frequency of paper coating cracking phenomena.

When folding the paper, a number of parameters must be considered in order to avoid damage of the paper coating, namely, the grammage of the base paper, the quantity of paper coating, the stiffness and the thickness of the end product, and the surrounding environment in which the paper finishing process has been performed, i.e., temperature, humidity and pressure on the calender. ${ }^{9-11}$

\section{EXPERIMENTAL \\ Materials}

Five different samples of label papers, taken from a production line of the paper mill Papirnica Vevče, 
were investigated, namely, PackPro 7.1 (in this study, denoted as $\mathrm{S} 1 ; \mathrm{G}=71.0 \mathrm{~g} / \mathrm{m}^{2}$ ), NiklaPack (S2;G = $\left.79.0 \mathrm{~g} / \mathrm{m}^{2}\right)$, NiklaSelect $8.0\left(\mathrm{~S} 3 ; \mathrm{G}=70.0 \mathrm{~g} / \mathrm{m}^{2}\right)$, NiklaPET $7.0\left(\mathrm{~S} 4 ; \mathrm{G}=70.0 \mathrm{~g} / \mathrm{m}^{2}\right)$ and PackPro 7.5 $\left(\mathrm{S} 5 ; \mathrm{G}=71.2 \mathrm{~g} / \mathrm{m}^{2}\right)$.

Paper samples were selected according to the tendency towards a higher quality of the end product and reduced complaints arising from phenomena of paper coating cracking. The examined coated label samples were selected as the most common paper products of the paper mill Papirnica Vevče. Specimens differed in terms of the raw material and the paper coating composition, but they all had in common oneside paper surface coating, i.e., the wire side, and were adequate for the lamination process.

At the paper mill, paper samples S1, S2 and S5 are intended for the production of flexible packaging, samples S3-S4 are meant for labels on non-returnable packaging, i.e., bottles, cans, jars, plastic packaging, etc. S1 and S5 are produced from wood-free cellulose fibers, while S2-S4 are produced from groundwood pulp. The studied label papers are suitable for the following printing techniques: offset, rotogravure and digital printing. ${ }^{13-17}$

\section{Methods}

The experimental methods were divided into two groups. In the first, the basic, surface and structural properties were studied (grammage, density, specific volume, moisture and ash content, roughness/porosity, contact measurement of roughness with a profilometer, tensile strength and elongation, and flexural and Clark stiffness).

In the second series, we examined the compositions of the coating blends and the base paper of the selected paper samples to determine the propensity towards cracking of the paper surface coating. Selected paper samples were further on offset printed with a black ink and folded under $1 \mathrm{~kg}$ load conditions for a period of 1 minute. This was followed by image analysis of the printed and folded samples. The images were captured using a Leica S9i optical microscope, preprocessed in Adobe Photoshop and analyzed with MathLab. All the tests carried out in the study were repeated on 10 specimens per sample.

\section{RESULTS AND DISCUSSION Microscopy and image analysis}

Figure 1 shows the area of the unfolded samples of the coated label papers studied. The paper samples are coated with a different amount defined as the sum of the precoat and the print coat from the paper mill production specifications. The smallest amount, i.e. 14.0 $\mathrm{g} / \mathrm{m}^{2}$, was applied to $\mathrm{S} 5$ and the largest - to $\mathrm{S} 2$, i.e. $20.7 \mathrm{~g} / \mathrm{m}^{2}$.

Figure 2 shows the degree of cracking of the paper coating after folding in machine direction
(MD) and in cross-direction (CD). Overall, the cracking of the paper coating is more pronounced in $\mathrm{MD}$, i.e., for all the paper samples (S1-S5) studied. The most obvious cracking of the paper coating after folding, i.e. in both directions (MD/CD) is seen in Figure 4 for $\mathrm{S} 3$ and the least obvious one - for S2 (Fig. 2). S5 shows a strong contrast between the cracking of the paper coating between MD and CD (Fig. 6), in CD the cracking of the paper coating being almost not visible.

The results regarding the extent of paper coating cracks were calculated using pixel differences between the images of the unfolded and folded paper samples, and are shown in Table 1. A comparison of the values from Table 1 reveals that the highest paper coating crack area (AC) is recorded for $\mathrm{S} 3$, i.e. $7.26 \%$, and slightly lower - for $\mathrm{S} 4$, i.e. $7.10 \%$. The paper sample with the lowest $\mathrm{AC}$ is S5, i.e. 5.30\%. Comparing the extent of cracking in the samples with the highest and the lowest values, there is a difference of $30.5 \%$.

The ratio of cracks to fibers is of $17.75 \%$, and the tendency of cracking in the paper coating is lower in $\mathrm{CD}$, i.e. $\mathrm{AC}-\mathrm{CD}=5.69 \%$, than in $\mathrm{MD}$, i.e. $\mathrm{AC}-\mathrm{MD}=6.91 \%$. The difference results from the influence of a pronounced anisotropy, which is reflected in the mechanical properties as a function of the direction of measurement, since the folding of the paper in MD leads to paper coating cracks perpendicular to the direction of fiber flow and parallel to the direction of bending. ${ }^{18}$ Despite the overall higher percentage of crack volume (CV) in MD, this observation does not apply without restriction to all the paper samples examined.

Paper coating cracking in $\mathrm{CD}$ on $\mathrm{S} 1$ and $\mathrm{S} 2$ is greater $(\triangle \mathrm{ACS} 1[\mathrm{CD} / \mathrm{MD}]=0.52 \%$; $\triangle \mathrm{ACS} 2[\mathrm{CD} / \mathrm{MD}]=2.13 \%)$ than on the other three samples, i.e., S3-S5. The largest variation in the cracking of the paper coating of the studied samples, according to the orientation of the cellulose fibers, can be observed in S3, where the difference is of $70.1 \%$ (Fig. 8). A comparison of the thickness value with the paper coating cracking data proves that the thinnest sample is the least susceptible to paper coating cracking, since the thickness of the paper is also affected by the amount of paper coating applied on the paper surface. The effect of the coating amount on the occurrence of paper coating cracking phenomena lies in the basic hypothesis that the amount of coating applied to the paper surface is proportional to the extent of occurrence of paper 
coating cracking. The amount of paper coating was determined as the sum of primer, i.e. PC, primer coating, and print coating, i.e. SC, second coating. S5 is a paper type exhibiting a high correlation to the amount of paper coating; it has the lowest amount of coating applied to the base paper, i.e. $14.0 \mathrm{~g} / \mathrm{m}^{2}$

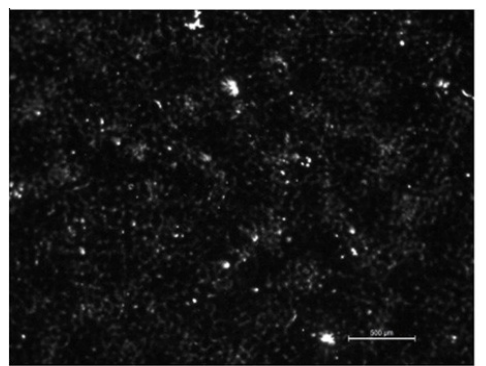

$\mathrm{S} 1$

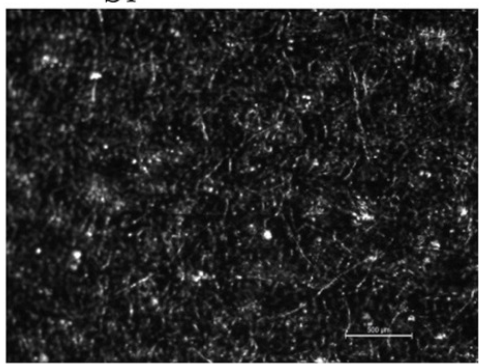

S4

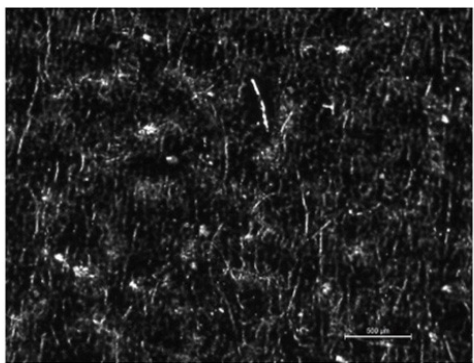

$\mathrm{S} 2$

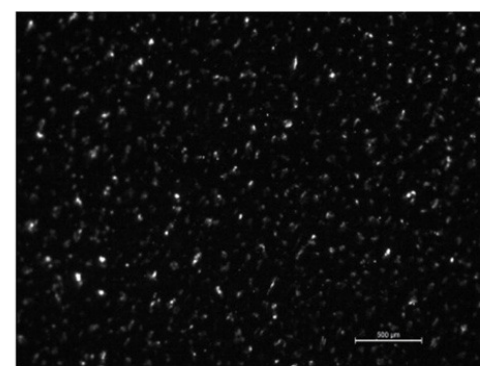

S3

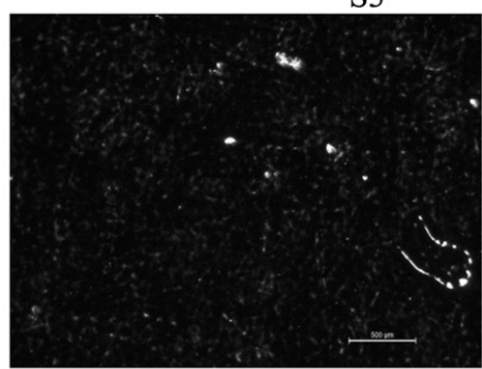

S5

Figure 1: Unfolded offset printed paper samples
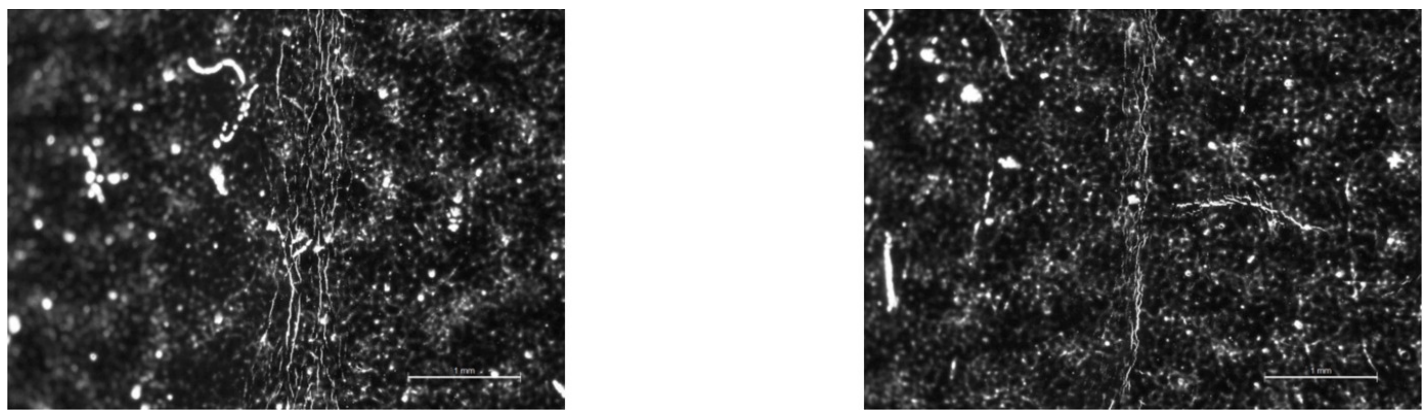

Figure 2: S1 - paper coating cracking after folding in MD and CD
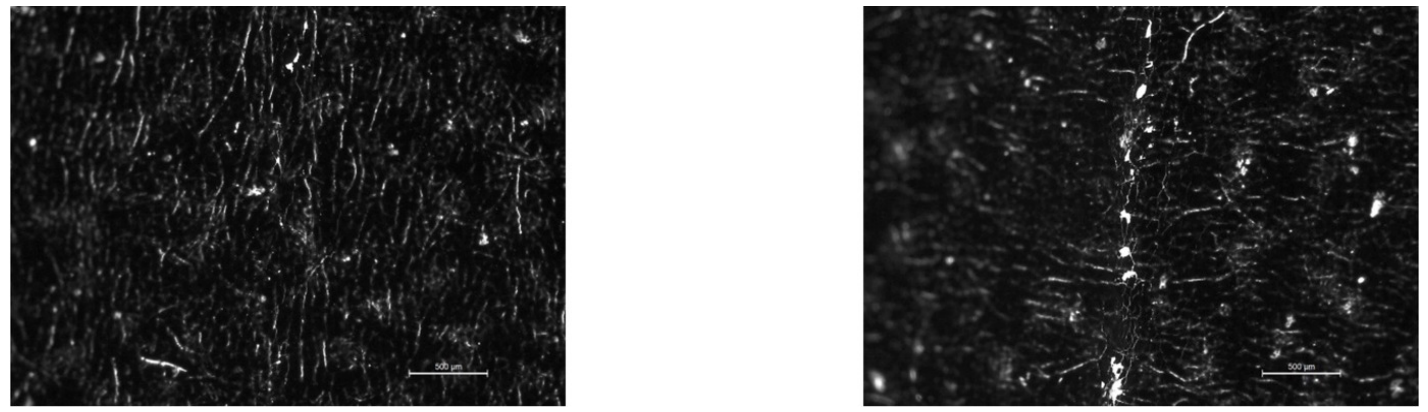

Figure 3: S2 - paper coating cracking after folding in MD and CD 

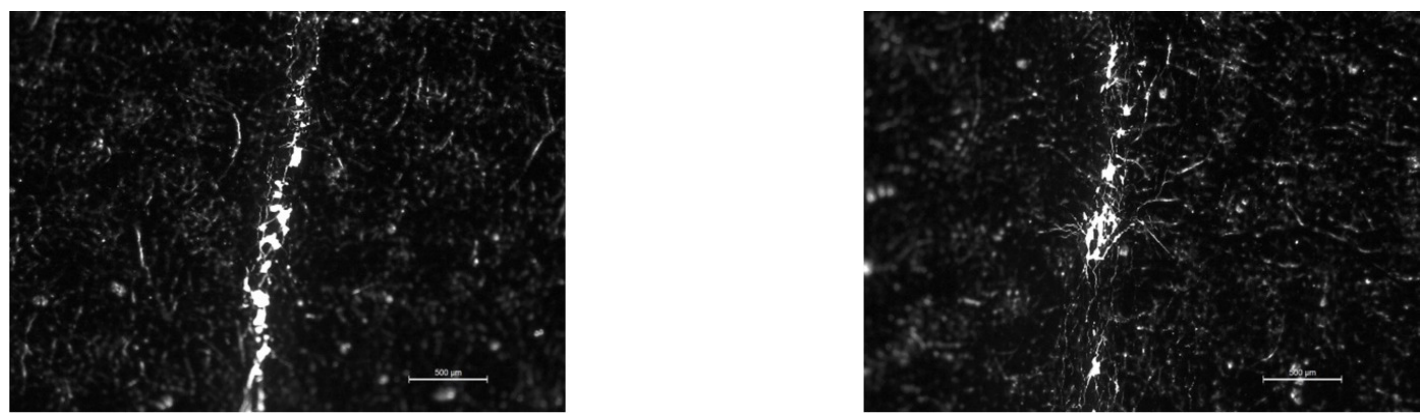

Figure 4: S3 - paper coating cracking after folding in MD and CD
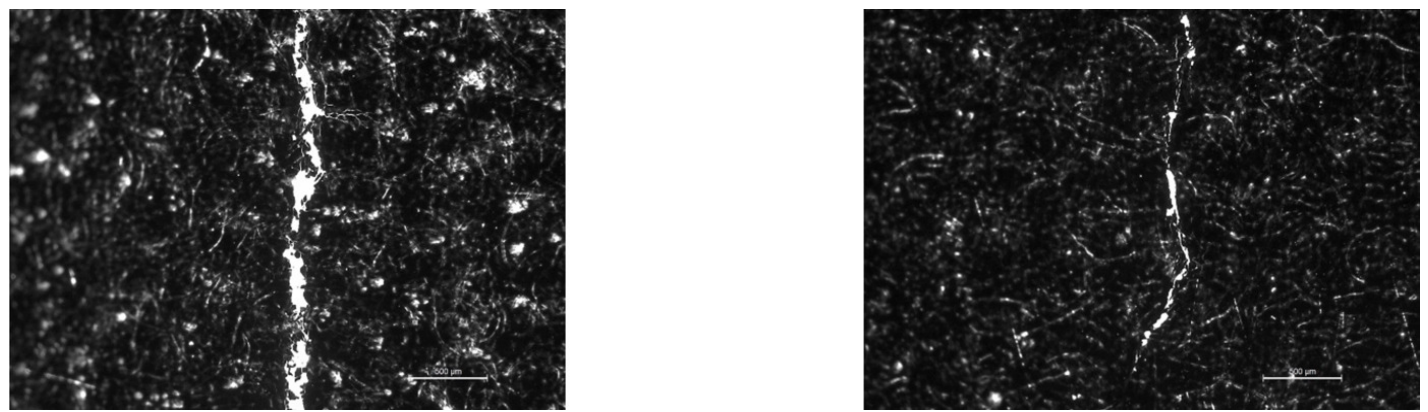

Figure 5: S4 - paper coating cracking after folding in MD and CD
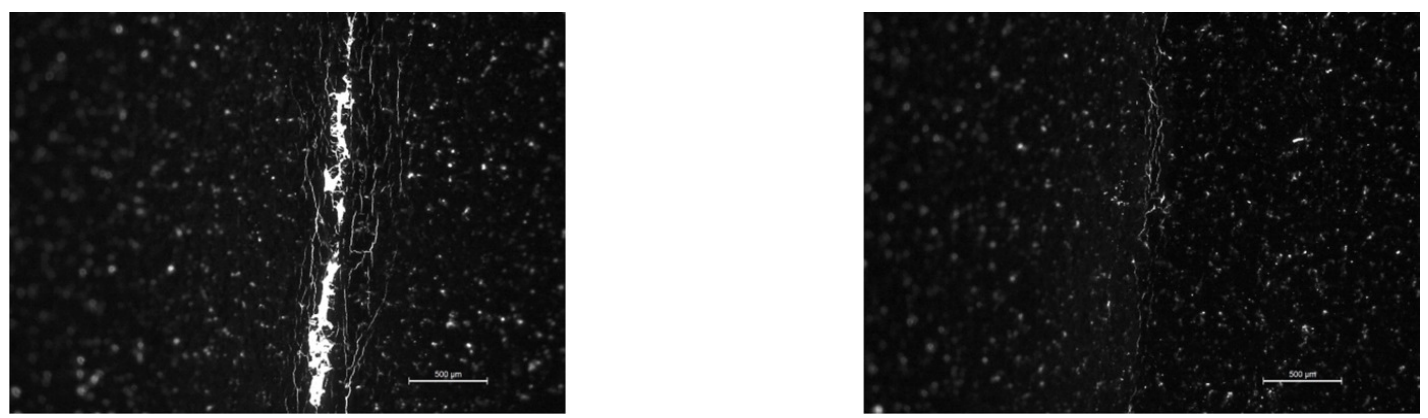

Figure 6: S5 - paper coating cracking after folding in MD and CD

Table 1

Extent of paper coating cracking

\begin{tabular}{lccccc}
\hline & \multicolumn{5}{c}{ Area of paper surface coating crack (\%) } \\
\cline { 2 - 6 } & S1 & S2 & S3 & S4 & S5 \\
\hline CD & 6.07 & 6.74 & 7.17 & 6.03 & 2.44 \\
MD & 5.55 & 4.61 & 8.08 & 8.18 & 8.17 \\
AC & 5.81 & 5.68 & 7.26 & 7.10 & 5.30 \\
\hline
\end{tabular}

The ash content determines the proportion of inorganic materials in the paper, which can be as high as $40 \%$ for graphic papers. Inorganic fillers attach to the cellulosic fibers and reduce the bonding among the fibers and the interweaving by acting as a physical barrier. Weaker bonds among fibers lead to faster and more intense cracking, especially in papers that have lower strength, because their cellulose fibers are shorter and fewer in number. This leads to a more rapid breakage of the fibers and to a rupture of the links among the cellulose fibers in a macrofibrillar 
network. From the manufacturer's data, calcium carbonate $\mathrm{H} 60$ is present in the primer layer of all the samples, except S4, where only starch was used (Table 4). Coarse and/or fine kaolin is also present as pigment in S1-S5. For S2 and S5, only coarsely ground kaolin is used in SC, while for S1, S4 and S5, finely ground kaolin is used. Calcium carbonate and kaolin are used as a combination in the composition of the coating mixture to fill the gaps between the fibers. The result is a reduction in the naturally embossed surface of the paper. The ash content in the papers is found in the following order: S3 $(19.56 \%)<\mathrm{S} 5$ $(20.31 \%)<\mathrm{S} 2(21.17 \%)<\mathrm{S} 4(23.46 \%)<\mathrm{S} 1$ (24.24\%).

Figure 7 shows the results of the coating percentage on the paper and the amount of $\mathrm{CaCO}_{3}-\mathrm{H} 60$ in the coating mixture. It can be remarked that the $\mathrm{CaCO}_{3}-\mathrm{H} 60$ content in the coating had the greatest negative effect on the appearance and extent of cracking of the paper coating. S4 contains only starch in PC, which has the same negative influence on the appearance of the paper coating cracks, i.e. it becomes rigid and not flexible enough to withstand the stresses.

According to the distribution, S4 confirms the assumption that the amount of inorganic components in the coating has an influence on the extent of paper coating cracking, while for S1-S3, the correlation between the measured values cannot be confirmed to this extent. In particular, S3 stands out for its lowest ash content determined $(19.56 \%)$, while it shows the highest tendency towards paper coating cracking, namely in $\mathrm{MD}$ and $\mathrm{CD}$, which can be attributed to the coating composition of $\mathrm{S} 3$, i.e. it has the highest proportion of $\mathrm{H} 60$ in the PC, $83.85 \%$ (Table 4). A higher percentage of calcium carbonate H60 means a larger number of large carbonate particles, which has a negative effect on the extent of cracking in the paper coating. To reduce the extent of cracking in the paper coating, it is more advisable to replace the $\mathrm{H} 60$ with an $\mathrm{H} 90$, since the latter has smaller and finer $\mathrm{CaCO}_{3}$ particles, which are more likely to be evenly distributed on the paper surface, giving it a more homogeneous structure, and therefore making it subject to a lower extent of cracking.

Kaolin is a pigment with flat-shaped particles and is a favorable choice when it comes to avoiding physical deformation, i.e. bending in the axial direction, and preventing paper coating cracks. A higher proportion of coarse kaolin, i.e., larger particles with a flat surface in the coating mixture applied to S3, means an increase in the risk of bending perpendicular to the axis of the particles and, consequently, a higher probability of the occurrence of paper coating phenomena. ${ }^{19-}$

${ }^{23}$ Both natural (starch) and synthetic (styrenebutadiene-latex) binders were used in all the paper coating mixtures, except in the PC for $\mathrm{S} 3$, where only starch was used (Table 4).

In Table 2, the samples are arranged according to the content of the binders (latex and starch) and pigments (H60 and H90) studied, from the lowest to the highest amount in the composition of the paper coating mixture.

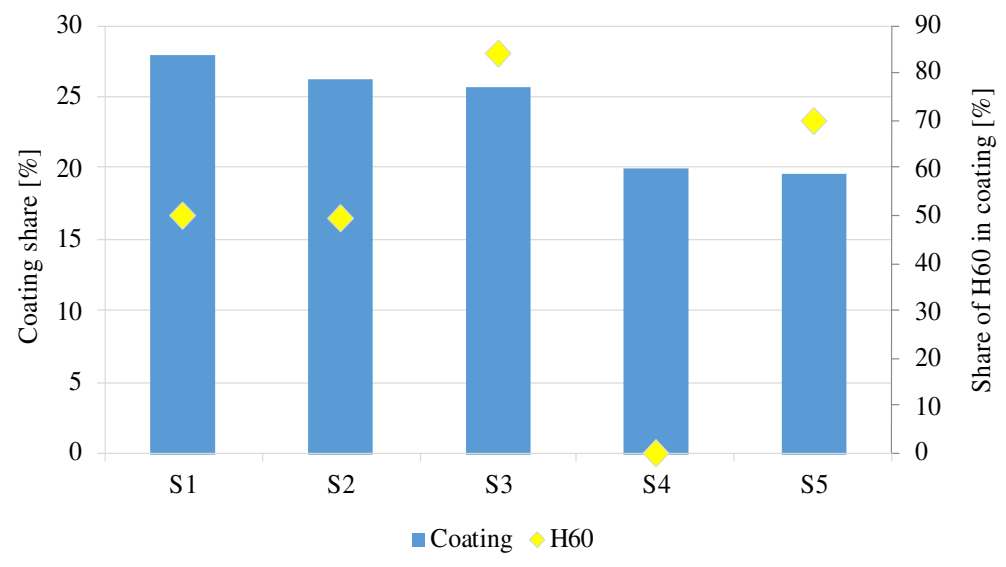

Figure 7: Share of coating applied on paper surface and amount of $\mathrm{CaCO}_{3}-\mathrm{H} 60$ in coating 
Table 2

Arrangement of samples according to the content of pigments and binders

\begin{tabular}{ccccc}
\hline & H60 & H90 & Latex & Starch \\
\hline 1. & S1 = S2 & S1 = S5 & S4 & S1 \\
2. & S5 & S2 & S5 & S2 = S3 \\
3. & S3 & S3 & S1 & S5 \\
4. & S4 & S4 & S3 & S4 \\
5. & $/$ & $/$ & S2 & $/$ \\
\hline
\end{tabular}

Table 3

Cellulose fiber ratio in base paper

\begin{tabular}{lccccc}
\hline \multicolumn{5}{c}{ Quantity of cellulose fibers $(\%)$} \\
\hline & Eucalyptus & Deciduous woods & Conifers & BCTMP & Fresh filler \\
\hline S1 & 32 & $/$ & 36 & $/$ & 0.0 \\
S2 & 25 & $/$ & 25 & $/$ & 0.0 \\
S3 & 37 & $/$ & 23 & 5 & 1.0 \\
S4 & 20 & 19 & 22 & $/$ & 0.6 \\
S5 & 37 & 40 & 23 & $/$ & 0.0 \\
\hline
\end{tabular}

Table 4

Share of significant components in paper coating mixture

\begin{tabular}{lccccc}
\hline & \multicolumn{3}{c}{ Film press (\%) } & \multicolumn{2}{c}{ Blade coater (\%) } \\
\hline & Starch & H60 & Kaolin & H90 & Kaolin \\
\hline S1 & 6.67 & 50.05 & 33.37 & 25.72 & 60.02 \\
S2 & 7.39 & 49.24 & 32.83 & 30.05 & 55.81 \\
S3 & 7.34 & 83.85 & 32,83 & 29.05 & 58.11 \\
S4 & 100.00 & 0.00 & 0.00 & 69.80 & 17.45 \\
S5 & 17.42 & 69.68 & 0.00 & 26.00 & 60.67 \\
\hline
\end{tabular}

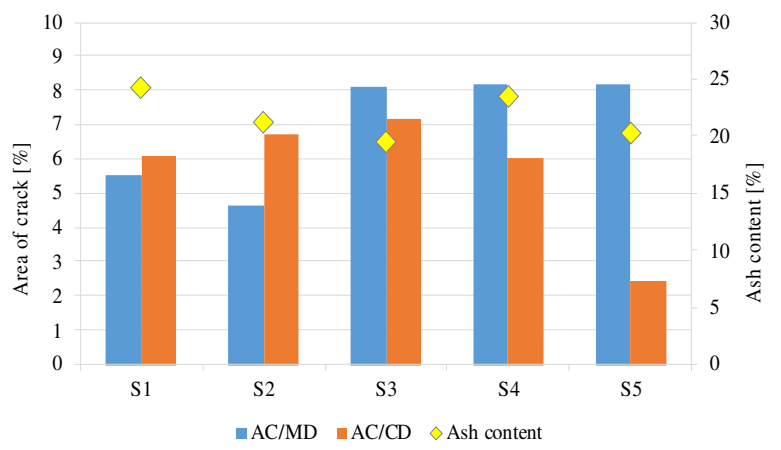

Figure 8: Area of paper surface coating crack and ash content

In the coatings of the studied samples of label papers, starch is present in all the mixtures, but in different amounts, of the primer coat. S4 contains the highest value of the primer coat $(8.72 \%)$, which also means the highest tendency of the paper coating to crack. This could be caused by the high content of natural binder, i.e. starch, which has a hard, film-forming property, with lower elasticity capacity, and is more prone to

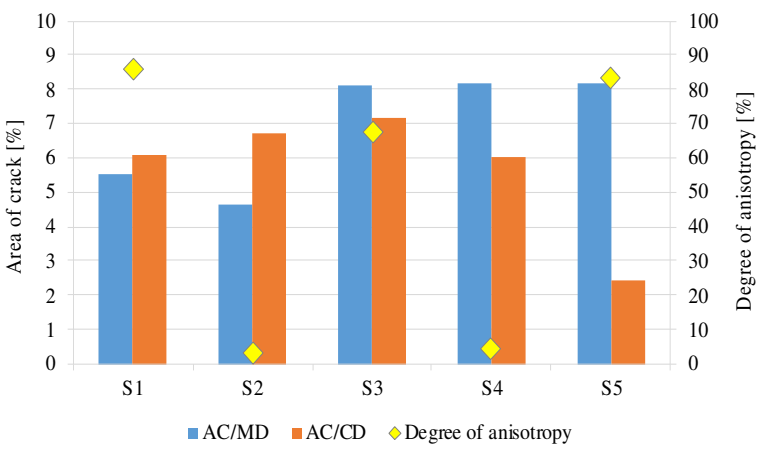

Figure 9: Area of paper surface coating crack and degree of anisotropy

cracking in the dry state than latex. Latex is, therefore, an infallible binder in modern paper production plants, with its distinct viscoelastic properties helping to improve stiffness, as a response to tension and elongation, i.e. to mechanical stress. ${ }^{24-26}$

The low starch content results in low cracking tendency of the paper coating, as measured in S1 and S2. The starch content in the paper coating of 
$\mathrm{S} 1-\mathrm{S} 3$ and S5 is lower than that of S4, i.e. 80$92 \%$. Coated papers containing higher percentage of latex are more flexible and stiffer, which is confirmed by the agreement between the coating crack volume data listed in Table 2 and the latex content in S2-S4. S5 is the sample with the lowest latex content, but at the same time, it is the most resistant to paper coating cracking phenomena $(5.30 \%)$, while S3 has the highest latex content $(16.32 \%)$ and the lowest resistance to paper coating cracking $(7.62 \%)$ among the studied paper samples. Coated paper is a composite material and each of the components contributes to the overall behavior of the material under physical and mechanical stress. In the case of S3, despite the high content of highly elastic latex, the cracking of the paper coating is more influenced by the higher amounts of $\mathrm{CaCO}_{3}-\mathrm{H} 60$ and coarse kaolin present in the coating blend in the largest amount among the studied paper samples, i.e. $\mathrm{S} 3 \mathrm{CaCO}_{3}-\mathrm{H} 60[\mathrm{PC}]=83.85 \%$ and coarse kaolin $[\mathrm{SC}]=29.05 \%$ (Table 4).

Figure 9 shows the relationship between the degree of anisotropy and the extent of cracking in the paper coating. Anisotropy is a change in physical properties as a result of the differential orientation of cellulose fibers in the paper fibril structure. Anisotropic properties directly affect the shrinkage and elongation at break of the paper, i.e., the elasticity of the paper. ${ }^{27,28}$ As shown in Figure 9, S1 $(85.71 \%)$ and S5 $(83.04 \%)$ have the highest degree of anisotropy. S2 (3.17\%) and S4 (4.65\%) have much lower anisotropic properties. A high degree of anisotropy means that the orientation of the fibers is predominantly in the MD direction, which affects the bending properties due to the difference in fiber orientation. ${ }^{29}$ In the studied paper samples, the influence of anisotropy can be seen in S2, which has a low paper coating cracking rate and low anisotropy. S3 has a relatively high degree of anisotropy and also exhibits the highest tendency for paper line cracking (7.62\%) under load.

When comparing the raw material composition of the base paper (Table 3) and the extent of coating cracking (Table 1), the influence of individual raw material contents on the tendency of paper coating cracking was found. The paper industry mainly uses hardwoods (eucalyptus, birch, poplar and beech) and softwoods (spruce, pine and fir) for paper production. The correct ratio between short (hardwood) and long (softwood) fibers, their fibrillation, as well as the sanding and fillers, are decisive for the quality of the end product. The loading behavior of the paper depends mainly on the quality and quantity of cellulose fibers, and the parameters of the paper machine must also be taken into account. ${ }^{30-}$

All the paper samples studied contain deciduous fibers, i.e. S1-S3 contain only eucalyptus fibers and conifers. S5 has the optimal formulation, in terms of paper coating appearance, while at the same time, containing the highest total content of broadleaf cellulose fibers $\left(\sum\right.$ (broadleaf + eucalyptus $\left.)=77 \%\right)$. The interweaving of cellulose fibers is higher in hardwood, which leads to a higher elastic response to external physical and mechanical stresses, i.e. also to a higher buckling strength. The higher density of hardwood and the greater wall thickness of cellulose fibers provide a stronger structure and a better response to external stresses. ${ }^{33,34}$ The experimental results are also consistent with the theoretical starting points in other cases, as the S1 and S3 samples, which achieve a high degree of cracking in the paper coating, while containing a low proportion of hardwood cellulose fibers, which translates into a low resistance to mechanical stresses. Bleached chemical wood pulp is present only in S3, i.e. in the paper sample with the highest measured crack volume ( $\mathrm{AC}=7.62 \%$ ), amounting to $5 \%$. The effect on the appearance of cracking in the paper coating, in relation to the raw material composition, was most evident when comparing the use of fresh filler in the paper composition of the studied paper samples, where the correspondence between cracking and fresh filler was identical in order (S3 > S4 > S1, S2 and S5). Samples S3 and S4 are the only ones containing fresh filler ( $\mathrm{S} 3=1.0 \%$ and $\mathrm{S} 4=0.6 \%)$ and, at the same time, the samples in which cracking due to paper coating was observed most frequently (AC; $\mathrm{S} 3=7.62 \%$ and $\mathrm{S} 4=7.10 \%$ ), which is explained by the fact that the higher filler content worsens the flexural strength after coating.

\section{CONCLUSION}

In the selection of paper surface coatings, it is of major importance that the coating penetrates the paper structure and evens out the topographical relief of the paper surface, so that the final product is capable of handling the high printing speeds with optimum printing properties, i.e. printability and runnability. In this study, papers with different coating formulations were evaluated in terms of their resistance to paper coating cracking. Label papers are one of the 
remarkable paper products that are gaining importance year by year, due to the everincreasing importance of the packaging industry. As the label paper undergoes a finishing process, it is also folded. The printed product must meet the high expectations of the customers and be of the highest possible quality. Printing ink, especially black ink, is the most reproducible ink, as it immediately indicates any irregularities in the paper composition. From the findings of the study, it may be concluded that, when $\mathrm{CaCO}_{3}$ is used in a paper coating formulation, H60 should be totally replaced, or at least partially substituted by $\mathrm{H} 90$ in a sustainable, i.e. economic, percentage, to avoid extensive cracking in the paper coating and thus, market complaints.

\section{REFERENCES}

1 K. Možina, $\mathrm{PhD}$ Thesis, University of Ljubljana, Faculty of Natural Sciences and Engineering, Ljubljana, Slovenia, 2017, pp. 10-23, https://repozitorij.unilj.si/IzpisGradiva.php?id=92705\&lang=slv

2 M. Larsson, G. Engström, D. Vidal and X. Zou, Nord. Pulp Pap. Res. J., 22, 267 (2007), https://doi.org/10.3183/npprj-2007-22-02-p267-274

3 Y. Wiputri and P. Englezos, Recent Pat. Eng., 1, 177

https://doi.org/10.2174/187221207780832138

(2007),

4 S. Wang, P. Ihalainen, J. Järenström and J. Peltonen, J. Disper. Sci. Technol., 30, 961 (2009), https://doi.org/10.1080/01932690802646447

5 U. Forsström, PhD Thesis, Helsinki University of Technology, Espoo, Finland, 2003, pp. 27-29, https://aaltodoc2.org.aalto.fi/bitstream/handle/1234567 89/2190/isbn9512269112.pdf?sequence=1\&isAllowed $=\mathrm{y}$

K. Možina, J. Ahtik, Š. Bečaj, U. Kranjc, M. Pivar et al., in Procs. $8^{\text {th }}$ Conference on Information and Graphic Arts Technology, Ljubljana, Slovenia, June 78, 2018, pp. 69-71, https://www.ntf.uni-lj.si/igt/wpcontent/uploads/sites/8/2018/06/8_CIGT_Proceedings. pdf

H. J. Youn, K. Sim, K. D. Oh, H. L. Lee, C. S. Han et al., Nord. Pulp Pap. Res. J., 27, 445 (2012), https://doi.org/10.3183/npprj-2012-27-02-p445-450

8 R. Dickson and U. Forsström, Nord. Pulp Pap. Res. J., 17, 434 (2002), https://doi.org/10.3183/npprj-200217-04-p434-439

9 C. Barbier, PhD Thesis, KTH Royal Institute of Technology, Stockholm, Sweden, 2004, p. 330-338, http://kth.diva-

portal.org/smash/get/diva2:8519/FULLTEXT01.pdf

10 M. Parpaillon, G. Engström, I. Pettersson, I. Fineman, S. E. Svanson et al., J. Appl. Polym. Sci., 30,
581

(1085),

https://doi.org/10.1002/app.1985.070300211

11 K. Oh, D. Seo, H. Y. Youn, Y. M. Lee, S. U. Yeu et al., Nord. Pulp Pap. Res. J., 31, 347 (2016), https://doi.org/10.3183/npprj-2016-31-02-p347-353

12 M. Černič Letnar, S. Grkman and J. Vodopivec, Restaurator, 27, $\quad 46 \quad$ (2006), https://doi.org/10.1515/rest.2006.46

13 PackPro 7.1. Tehnical sheet. Available on-line: $<$ https://www.brigl-

bergmeister.com/sl/izdelek/packpro-7-1/>

14 NiklaPack. Tehnical sheet. Available on-line: $<$ https://www.brigl-

bergmeister.com/sl/izdelek/niklapack/>

15 NiklaSelect. Tehnical sheet. Available on-line: $<$ https://www.brigl-

bergmeister.com/sl/izdelek/niklaselect/>

16 NiklaPET. Tehnical sheet. Available on-line: https://www.brigl-bergmeister.com/sl/izdelek/niklapet/

17 PackPro 7.5. Tehnical sheet. Available on-line: $<$ https://www.brigl-

bergmeister.com/sl/izdelek/packpro-7-5/>

18 K. Oh, K. Sim, Y. B. Jung, H. J. Youn, H. L. Lee et al., Nord. Pulp Pap. Res. J., 30, 360 (2015), https://doi.org/10.3183/npprj-2015-30-02-p360-367

19 G. Novak, in "Graphical Materials", edited by D. Gregor Svetec, D. Dolenc and G. Golob, University of Ljubljana, Faculty of Natural Sciences and Engineering, Department of Textiles, 2004, pp. 95-110

20 P. A. Gane, J. P. Kettle, G. Peter Matthews and C. J. Ridgway, Ind. Eng. Chem. Res., 35, 1753 (1996), https://doi.org/10.1021/ie950413m

21 Y. Du, Y. H. Zang, and J. Du, Ind. Eng. Chem. Res., $\quad$ 50, $9781 \quad$ (2011), https://doi.org/10.1021/ie200807w

22 S. Tyagi, A. K. Ray and Y. V. Sood, J. Coat. Technol. Res., 7, 747 (2010), https://doi.org/10.1007/s11998-010-9261-6

${ }^{23}$ Z. Hu, X. Zen, J. Gong and Y. Deng, Colloid.

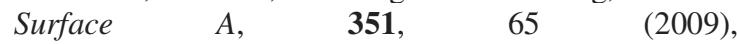
https://doi.org/10.1016/j.colsurfa.2009.09.036

24 A. Hedman, M. Wikström and M. Rigdahl, Nord. Pulp Pap. Res. J., 16, $266 \quad$ (2001), https://doi.org/10.3183/npprj-2001-16-04-p266-273

25 R. J. Ashley, M. A. Cochran and K. W. Allen, Int. J. Adhes. Adhes., 15, $101 \quad$ (1995), https://doi.org/10.1016/0143-7496(95)98745-8

26 H. Maruyama, K. Kajitani and M. Shiraishi, U.S. Patent No. 4617239A, 1986, 8 p., https://patentimages.storage.googleapis.com/94/03/e5/ d482b00e5ea99c/US4617239.pdf

27 A. Kulachenko and T. Uesaka, Mech. Mater., 51, 1 (2012), https://doi.org/10.1016/j.mechmat.2012.03.010 28 A. Kulachenko, S. Lindström and T. Uesaka, in Procs. Papermaking Research Symposium, Kuopio, Finland, June, 1-4 2009, pp. 35-45 
S. Lavrykov, S. B. Lindström, K. M. Singh and B. V. Ramarao, Nord. Pulp Pap. Res. J., 27, 256 (2012), https://doi.org/10.3183/npprj-2012-27-02-p256-263

30 M. Černič, "Quality of Graphic Papers in Digital Printing Techniques: Expert Study”, Pulp and Paper Institute, Ljubljana, Slovenia, 2017, pp. 4-34

31 M. Alava and K. Niskanen, Rep. Prog. Phys., 69, $669 \quad$ (2006), https://doi.org/10.1088/0034$4885 / 69 / 3 / \mathrm{r} 03$

32 K. Niskanen and P. Pakarinen, in "Paper Structure, Papermaking Science and Technology", Book 16, Paper Physics, $2^{\text {nd }}$ ed., edited by K. Niskanen, Fapet Oy, Helsinki, Finland, 2008, pp. 11-58

33 B. Iglič, Short technology of fiber extraction and production of paper and cardboard for woodworking students at the Biotechnical Faculty of Edvard Kardelj: invited lecture. University of Ljubljana, Biotechnical Faculty, 1988, pp. 9-11, http://sylviabmiller.me/download/pIApNAAACAAJkratka-tehnologija-pridobivanja-vlaknin-inproizvodnja-papirja-ter-kartona-za-slusatelje-lesarstvana-biotehniski-fakulteti-univerze-edvarda-kardelja-vljubljani

34 W. W. Sampson, Int. Mater. Rev., 54, 134 (2009), https://doi.org/10.1179/174328009x411154 